Escuela de Ciencias Sociales y Humanidades, UNED, C.R.

URL: http://investiga.uned.ac.cr/revistas/index.php/espiga/index

ISSN: 1409-4002 • e-ISSN: 2215-454X

Doi: http://dx.doi.org/10.22458/re.v17i35.2093

\title{
Aprendiendo para el futuro: una experiencia en Finlandia
}

\author{
Ileana Garita-González* \\ iD https://orcid.org/0000-0001-5330-0566
}

Recibido: 18 de mayo, 2018 • Aceptado: 06 de junio, 2018

\section{RESUMEN}

En las últimas décadas, Finlandia apostó por un modelo educativo del más alto nivel. De esta experiencia exitosa se ha escrito en reiteradas oportunidades, pero normalmente enfocada en la educación y metodología aplicada en el periodo que ellos llaman «Escuela comprensiva», que se extiende por diez años a partir de que el niño cumple los seis años de edad.

En este artículo se aborda una experiencia práctica a través de la participación en la «Semana Internacional de Savonia UAS 2018», evento que reúne a connotados profesores universitarios y funcionarios de diversas universidades del mundo, con gran participación de universidades europeas. En el marco de dicho evento y actividades posteriores realizadas, se pretende conocer un modelo de educación universitaria desde la concepción de la Facultad de Negocios, Turismo y Hospitalidad de la Universidad de Ciencias Aplicadas de Savonia (UAS) y las posibilidades que se brindan de identificar estrategias de enseñanza innovadoras que pueden ser aplicadas en la carrera de Gestión Turística Sostenible de la Universidad Estatal a Distancia (UNED) y las posibilidades futuras de cooperación interuniversitaria.

Palabras clave: Finlandia, educación, turismo, innovación.

\section{Formato de citación según APA}

Garita-González, I. (2018). Aprendiendo para el futuro: una experiencia en Finlandia. Revista Espiga 17(35), 106-115. Doi: http://dx.doi.org/10.22458/re.v17i35.2093

\section{Formato de citación según Chicago-Deusto}

Garita-González, Ileana. «Aprendiendo para el futuro: una experiencia en Finlandia». Revista Espiga 17, n. ${ }^{\circ} 35$ (junio, 2018): 106-115. Doi: http://dx.doi.org/10.22458/ re.v17i35.2093

Máster en Desarrollo Económico Local por la Facultad Latinoamericana de Ciencias Sociales (FLACSO) y el Instituto Tecnológico de Costa Rica (ITCR). Gestora de Turismo Sostenible por el ITCR. Licenciada en Derecho por la Universidad Florencio del Castillo; especialista en Derecho Notarial por la Universidad de Costa Rica. Consultora nacional e internacional. Coordinadora de la Carrera de Gestión Turística Sostenible y de la licenciatura en Gestión y Gerencia del Turismo Sostenible de la Escuela de Ciencias Sociales y Humanidades de la Universidad Estatal a Distancia (UNED). Docente en grado de la UNED, Costa Rica. Correo: igarita@uned.ac.cr 
Dentro del quehacer institucional, la Universidad Estatal a Distancia (UNED) genera espacios que favorecen el intercambio de conocimientos, un ejemplo de ello fue la firma de una "Carta de Entendimiento» con la Universidad de Ciencias Aplicadas de Savonia (UAS). Los primeros acercamientos se originan a través de la Escuela de Administración de Negocios, pero rápidamente se extienden a otras instancias y se genera una expectativa de cooperación entre la Facultad de Negocios, Turismo y Hospitalidad de dicha universidad y la Carrera de Gestión Turística Sostenible de la Escuela de Ciencias Sociales de la UNED.

Dentro de este proceso se concibe la participación de un integrante del equipo académico de la carrera de Gestión Turística Sostenible, en el evento denominado «Semana Internacional de Savonia UAS 2018», que a su vez incluye el desarrollo de la actividad «Open Innovation Space (OIS 2.0)»; ambos realizados en la ciudad de Kuopio, Finlandia, en febrero de 2018.

La participación en los eventos en mención tuvo como propósito conocer y analizar la realidad educativa y académica de la oferta en el área del turismo y la hospitalidad y su ligamen con el área de negocios y asimilar las tendencias educativas entre estudiantes y docentes en general.

De igual manera, se fortalece la generación de puentes entre ambas universidades en función de la búsqueda de oportunidades para realizar acciones en el ámbito de la innovación y la generación de experiencias relacionadas con la vinculación universidadempresa, aspecto en el que la UAS tiene mucho camino recorrido.

La importancia de la participación en las actividades descritas radicó en la inigualable oportunidad de conocer y analizar la experiencia académica de educación superior en el área del turismo, y la posterior aplicación de estrategias de enseñanza orientadas a un mejoramiento de la carrera de Gestión Turística Sostenible que imparte la UNED.

\section{Finlandia y su sistema educativo}

El sistema educativo finlandés es catalogado como uno de los mejores del mundo. En él, la educación superior se ha convertido en un importante sector de la política educativa en el que se acentúan la investigación científica y la instrucción en tecnología de la información y la comunicación.

Por su parte, la UAS es una de las universidades de ciencias aplicadas más grande de Finlandia, que ofrece programas de licenciatura y maestría en diversas áreas, incluyendo varios énfasis relacionados con turismo y hospitalidad, lo que supone posibilidades de proyectos conjuntos en diferentes campos.

En el sector de interés para este artículo, una parte de estas carreras relacionadas con Turismo y Hospitalidad se puede desarrollar bajo un dinámica «semipresencial», que presenta gran similitud con el modelo a distancia de la UNED, con la utilización de componentes tecnológicos para desarrollarla.

Desde el 2010, la UAS viene trabajando bajo el Modelo Pedagógico «Open Innovation Space (OIS 2.0)», que radica en un cambio en la forma de pensar y busca conectar 
la educación avanzada con las prácticas de desarrollo, investigación e innovación orientadas a la vida laboral, mediante la asociación entre varios actores: representantes de empresas, profesores y estudiantes, donde se trabaja en diferentes tipos de tareas como parte de una visión de estudios multidisciplinarios.

Una de las mayores desventajas que se han identificado en la población universitaria de la UNED, precisamente es la vinculación orientada a la integración de los estudiantes en el ámbito laboral. Este y otros aspectos abordados en el plan de mejora que se sigue en la carrera, serán los aspectos que se busca optimizar, analizando perspectivas educativas novedosas.

\section{Modelo educativo finlandés}

Finlandia apostó por el fortalecimiento de su sistema educativo, mismo que se concibe como uno de sus pilares; por consiguiente, es entendible que en este país la educación es gratuita en todas sus etapas.

El modelo de educación en Finlandia se desarrolla desde la primera infancia, pero no es hasta los seis años que inicia la educación formal durante diez años ${ }^{1}$, divididos en varias etapas. Este periodo se conoce como «Escuela comprensiva o incluyente», según mencionó Jouni Valijarvi ${ }^{2}$ en una conferencia dictada sobre «La evaluación y pedagogía en la Escuela comprensiva de Finlandia», impartida en Bogotá, Colombia en 2008:

«(...) nosotros tenemos en Finlandia lo que se conoce como la Escuela comprensiva o incluyente; nosotros nos dedicamos a desarrollar este sistema desde los años1970. En este momento tenemos un sistema escolar de nueve años de educación obligatorios para todos los jóvenes, y esto quiere decir además que nosotros no hacemos ningún tipo de seguimiento en estas escuelas incluyentes a comparación de muchos otros países europeos como Europa Central [sic] vemos que nuestro sistema es visto como algo realmente excepcional. Ahora el concepto básico de las escuelas incluyentes es que todos los estudiantes, todo tipo de estudiantes trabajan en los mismos grupos con un solo docente. Es decir, que un solo o sola docente se encarga de todos los estudiantes de su grupo, incluyendo todas las diversidades y diferencias, y eso también es cierto para los años superiores, o lo que nosotros llamamos la escuela media, que empieza en el grado séptimo. Es cuando los estudiantes empiezan a aprender a trabajar juntos. Yo diría que esa es una de las filosofías más importantes de este sistema finlandés y tiene mucho que ver con cómo manejar la heterogeneidad de los estudiantes y cómo utilizar la evaluación para apoyar a todos los estudiantes que conforman esos grupos heterogéneos en una escuela comprensiva. Entonces, esta escuela incluyente realmente es integral, implica que todos los estudiantes van a recibir el mismo programa, el mismo currículo; sin embargo existe la posibilidad de tomar decisiones individuales y de hacer elecciones personalizadas, pero se trata de decisiones muy limitadas comparadas con la totalidad del programa o currículo.»

1. Durante la estadía en la ciudad de Kuopio, en Finlandia, en reiteradas ocasiones se sitúa la escuela comprensiva o incluyente con una duración de diez años; no obstante, el autor Jouni Valijarvi menciona que se desarrolla durante nueve años. Estos datos presentan un desfase, lo cual no es un error, es solo la inclusión de datos suministrados en dos momentos distintos, uno en forma presencial durante una presentación universitaria y otro bibliográfico.

2. Profesor de la Universidad de Jyväskylä, Finlandia. 
Luego, los estudiantes pueden elegir seguir entre la escuela secundaria superior o la escuela vocacional y el entrenamiento de aprendices. Al finalizar, en ambas opciones se debe realizar un examen de calificación (diferente de acuerdo con la escogencia).

En relación con la educación universitaria se presentan dos opciones: la universidad como tal (grados de bachillerato, maestría y doctorado) y la universidad politécnica (grados de bachillerato y maestría). Esta última opción puede obtener el grado universitario, equiparando las carreras y completando estudios.

Para una mejor comprensión, en la figura 1 se presenta en forma gráfica el sistema educativo finlandés.

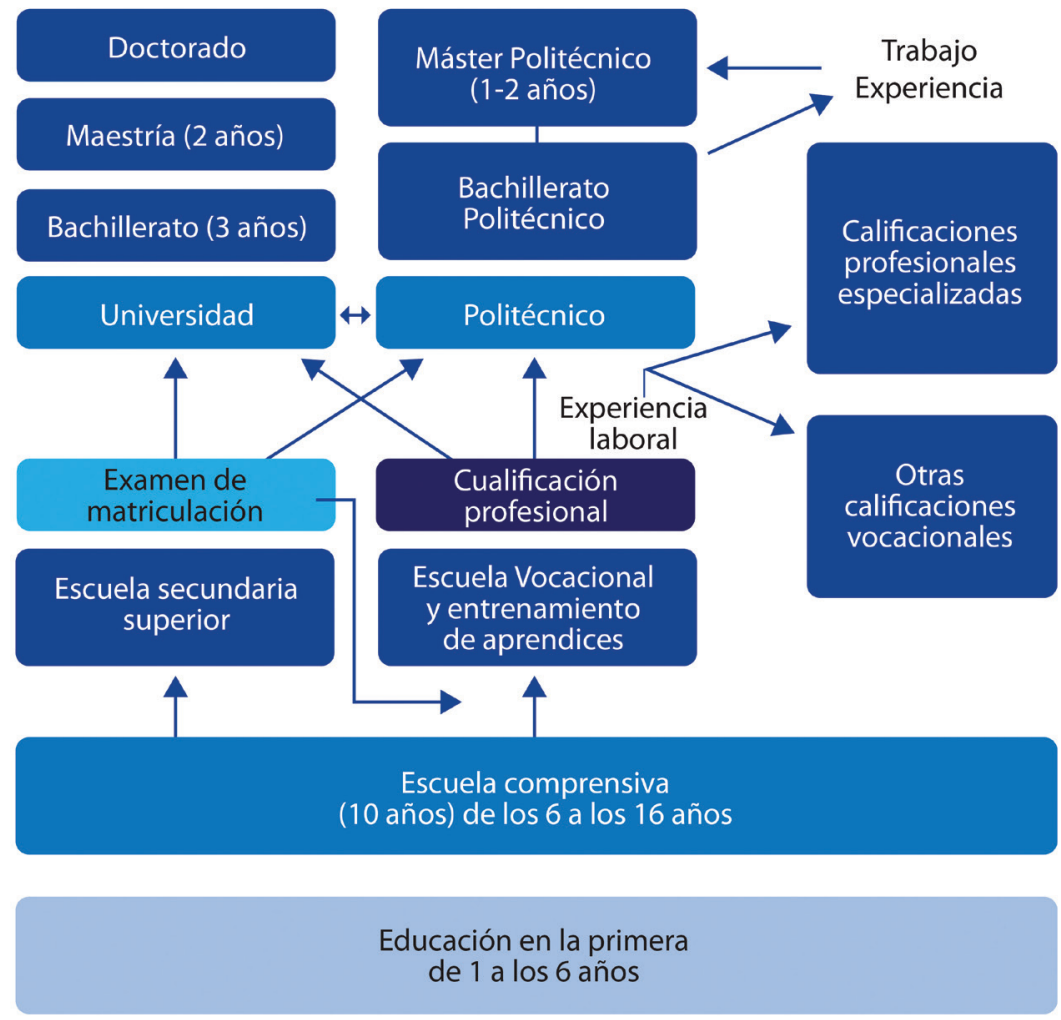

Figura 1. Sistema educativo finlandés.Fuente: Sitio web de Savonia University of Applied Sciences.

Específicamente en la carrera de Turismo y Hospitalidad, cada uno de los cursos que se imparten tiene asignado un equipo interdisciplinario ${ }^{3}$ de profesores, desde la planeación hasta el desarrollo (pueden ser pares, tres o más profesores).

El estudio de otras lenguas es básico. Dentro del diseño estructural de su plan de estudios se asignan diez créditos al estudio de idiomas y el estudiante puede escoger entre

3. En uno de los cursos analizados, la dinámica colaborativa observada fue la de siete profesionales de diferentes áreas, quienes trabajaban de manera conjunta. 
varias opciones; sin embargo, se insiste en la necesidad de aprender el idioma ruso, por ser su principal mercado turístico. De igual manera el inglés está inserto dentro del plan de estudios, más de la mitad de los cursos se imparten en inglés y uno de los énfasis de la carrera de Turismo y Hospitalidad solamente se imparte en inglés. Los profesores se certifican con el modelo de la Universidad de Oxfort, lo que les brinda la calidad para impartir los cursos.

La UAS cuenta con un departamento de negocios, el cual se encarga de contactar empresas o en algunos casos las empresas contactan a la universidad, con miras a la obtención de espacios para desarrollo de proyectos donde participan los estudiantes, pero también como posibles empleadores del estudiantado.

Las actividades son variadas, planificadas por el profesor o profesores que imparten el curso: exámenes, especialmente en las materias básicas, y proyectos de investigación en casi todas las asignaturas de los años superiores.

\section{Semana Internacional de Savonia UAS 2018}

La Semana Internacional de Savonia UAS 2018 (SIS UAS-2018) es un evento anual que reúne a profesores y funcionarios de muchos países interesados en conocer el modelo de enseñanza universitaria finlandés y sus técnicas de aprendizaje y en crear lazos de cooperación entre universidades. Este año se llevó a cabo entre el 19 y el 23 de febrero e involucró la participación en múltiples actividades.

El tema de SIS UAS-2018 fue «Impact Creation Day» (la creación de un impacto diario). Los principales aspectos fueron educar para que los estudiantes tengan un impacto futuro y hagan de su región y país un lugar exitoso, y la creación de conexiones para hacer un cambio en el futuro.

La actividad inaugural analizó el tema «Learning for the future» desde cuatro aspectos.

1. Lo primero que se indica es que «el futuro es ahora», donde se señala que los cambios actuales son sumamente acelerados, continuamente se descubren o materializan nuevos productos, nuevas tecnologías, prácticamente año a año, mes a mes e incluso día a día. Los grandes cambios se están produciendo rápidamente, por lo que adivinar cuál tecnología se usará en el futuro es un reto. Lo que se mantiene desde épocas antiguas es el ser humano y su interés por conocer cosas nuevas.

Hoy se hacen cosas para ir a un trabajo que posiblemente no exista en el futuro; la historia demuestra cómo muchos trabajos se van evaporando con el tiempo (Revolución Industrial, Revolución Tecnológica, tiempos modernos). Un ejemplo local fue la reciente puesta en funcionamiento de un autobús-robot en la capital Helsinki, por lo que en un futuro cercano es posible que ya no haya choferes de autobús.

2. Como segundo elemento se presenta la consigna «predecir el futuro es imposible». El futuro es impredecible y la mejor forma para predecir el futuro es creando; cuando se crea se es parte de la innovación. En educación se menciona que aprender puede ser divertido si estimulamos en los estudiantes la curiosidad y mejoramos su capacidad de investigación. 
3. También se comprende que «aprender es un trabajo duro». Aprender puede ser divertido, lo que no significa que sea fácil (divertido no es igual que fácil). Para aprender se necesitan buenos maestros, dedicar tiempo y esfuerzo y brindar al estudiante la adecuada motivación para desarrollar nuevas capacidades. Muchas veces la frustración puede matar la creatividad para hacer cosas nuevas.

4. Por último, se profundiza sobre que «aprender es un puente del conocimiento», pero no solo se aprende a través del estudio académico; la vida misma, las vivencias diarias nos enseñan, se aprende en cada uno de los momentos, las experiencias generan un valioso aprendizaje.

\section{Otras actividades}

Dentro de las actividades de la SIS UAS-2018 se llevó a cabo el «Discover World», que consistió en la exposición y presentación de información de los países en los que estudiantes de la UAS han tenido o están planificando intercambios de movilidad estudiantil. Al pertenecer Finlandia a la zona Schengen (área que comprende a 26 países europeos que han abolido los controles fronterizos), la posibilidad de sus estudiantes de realizar estudios o especializaciones en otros países que integran la misma zona es sumamente alta.

Los estudiantes de la UAS que participaron en este evento, conocieron la experiencia de los estudiantes que han desarrollado o están en proceso de desarrollar actividades de movilidad estudiantil y promoviendo los países anfitriones.

Costa Rica estuvo representada por un estudiante de la UAS que posteriormente realizaría una pasantía en el país, específicamente para realizar su práctica de graduación, por lo que se logró el objetivo de dar a conocer el país y a la UNED como posibilidad de movilidad laboral.

\section{Open Innovation Space}

En Savonia, la educación de UAS se basa en un concepto llamado OIS, que son las siglas en inglés de «Open Innovation Space». El concepto combina el aprendizaje orientado al trabajo y las actividades de investigación y desarrollo que lo respaldan.

Durante la SIS UAS-2018 se realizó la presentación al público de los proyectos desarrollados por los estudiantes para este concepto dentro de una feria que lleva el mismo nombre «Open Innovation Space». En ella, participan diversos grupos de estudiantes que comparten con el público en general sus proyectos, simulan una feria de negocios y venden al público su producto o servicio y hasta se simula la captación de inversores. Al final del día hay una premiación para los proyectos más innovadores, mejor trabajados y que lograron un mayor número de inversores.

Para explicar este enfoque, se puede decir que radica en la creación y profundización de las conexiones entre los estudiantes y la vida laboral, de modo que sea posible que cada estudiante se familiarice con los trabajos y las tareas en un campo particular. 
Se desarrollan proyectos en diversos campos de estudio donde se aplican métodos específicos para implementar formas de aprendizaje activas, experienciales y orientadas a la vida laboral. Un ejemplo es el marco CDIO (Conceive-Design-Implement-Operate), que se utiliza en ingeniería y tecnología.

La educación orientada a la vida laboral en la UAS se hace posible gracias a las versátiles redes que el personal docente ha creado; este personal es experto en planificar el proceso de aprendizaje y apoyar el desarrollo de sus estudiantes.

El estudiantado, por su parte, son estudiantes activos que asumen la responsabilidad de su progreso. Trabajar en varios grupos y entornos de aprendizaje es una parte natural de los estudios, donde estudiantes, profesores, personal de I+D y representantes de diversos campos resuelven problemas juntos, y esto requiere un conocimiento interdisciplinario.

Así es como los estudios combinan teoría y práctica. Los estudios más teóricos se apoyan en un fuerte vínculo con la vida laboral, que se hace evidente cuando los estudiantes realizan su pasantía y trabajan en sus proyectos de tesis, así como en las tareas de desarrollo y proyectos encargados por la vida laboral.

Durante sus estudios, los estudiantes crean redes y conocen las empresas locales, lo cual es esencial para encontrar un empleo. Aquellos que aspiran a convertirse en empresarios pueden encontrar su base de clientes durante los estudios. Con la ayuda de una estrecha cooperación con las empresas, los estudiantes conocen a las empresas locales y los grupos de interés e identifican las oportunidades que ofrecen, sin importar cuál sea el objetivo: el espíritu empresarial o trabajar para otra persona.

\section{Tecnología aplicada a la educación}

La UAS cuenta con tecnología de punta para la enseñanza de las carreras que imparte. Algunos ejemplos de estas herramientas son:

- Se apoya el aprendizaje de los estudiantes con la versión actual de la plataforma Moodle.

- Cuenta con espacios de simulación de situaciones propias de la práctica profesional.

- En el desarrollo de productos se cuenta con impresoras 3D, que crean el producto a partir de un sotfware.

- Utilizan el sistema de videoconferencias ZOOM con servicios en la nube; en los diferentes recintos universitarios tienen un total de 200 cámaras y micrófonos instalados.

- Mediante el portal de Movilidad Online, los estudiantes acceden fácilmente y hacen sus propias conexiones.

- Los servicios de biblioteca están totalmente automatizados, lo que facilita el servicio con poco personal. 
- Hay espacios privados con todos los implementos tecnológicos para que los estudiantes puedan desarrollar proyectos, reuniones o incluso exámenes sin que esté presente el profesor.

\section{Espacios prácticos en Turismo y Hospitalidad}

Con la metodología IOS, la carrera de Turismo y Hospitalidad involucra a los estudiantes directamente con la práctica de su especialidad a través de diferentes proyectos. Algunos ejemplos de estos proyectos en el ámbito del turismo son:

- Diseño e implementación de un restaurante emergente «Pop Up». Los estudiantes de primer año combinan los contenidos de tres cursos para practicar y aplicar conocimiento.

- En el marco del proyecto del Pop Up, se organizan y desarrollan varios eventos al año, como parte de los estudios.

- Estudio de proyectos denominado «Futuro Food». Los estudiantes realizan propuestas que desarrollan sobre productos del entorno alimentario del futuro y pueden realizar pruebas de los productos para su comercialización, así como de los servicios conexos.

- Involucramiento con el círculo empresarial como parte del aprendizaje de conocimientos sobre jurisprudencia empresarial y contractual.

- Visitas de estudio y participación de actores involucrados en el desarrollo de la región.

- Excursiones de estudio (feria «Gastromess», ferias de viajes, otras), que pueden ser nacionales o internacionales como parte de los cursos.

Hace 10 años se inició un programa piloto en Savonia para brindar apoyo a los estudiantes durante sus estudios, orientado al desarrollo de su propio negocio o emprendimiento. Para esto, se cuenta con un grupo de profesores que apoyan los emprendimientos estudiantiles con la idea de que los alumnos en cualquier campo o especialidad, puedan desarrollar sus ideas de negocios durante los años de estudio.

La iniciativa emprendedora significa que los estudiantes desarrollan sus productos durante los años académicos. Es una manera de ayudar a los estudiantes a desarrollar sus propias ideas. Además, es un modelo mental para cambiar la vida.

Desde que se instauró el proyecto se han inscrito más de 500 estudiantes en este programa, quienes deciden cuáles cursos pueden servir para su propia empresa e incluso pueden realizar la práctica de la carrera en su propia empresa.

\section{Lo que sigue}

La experiencia desarrollada durante la participación en la SIS UAS-2018, brinda a la carrera de Gestión Turística Sostenible de la UNED una gran oportunidad para generar proyectos conjuntos con la UAS. 
Ya se inició el intercambio estudiantil entre ambas universidades con la visita de uno de los estudiantes de la UAS y se espera en este mismo año se concrete la posibilidad de varios estudiantes de la UNED de desarrollar un proyecto o pasantía en Finlandia. De igual manera, existe gran interés por parte de la UAS en realizar intercambios que conlleven cursos virtuales, donde estudiantes de Savonia puedan participan en cursos UNED y viceversa.

También, se abre la posibilidad de implementar un modelo de desarrollo de emprendimientos en la UNED como el OIS desarrollado por Savonia como un plan piloto de involucramiento de estudiantes con empresas y/o proyectos institucionales.

Estos y otros proyectos se pueden desarrollar en el futuro, lo que brinda al cuerpo docente de la UNED y al estudiantado la posibilidad de integrar aspectos novedosos de un país como Finlandia en el modelo educativo que ofrece la UNED que puede enriquecer el conocimiento que se adquiere.

\section{SUMMARY}

\section{Learning for the future: a Finnish experience}

In the last decades, Finland opted for the highest educational model possible. Of this successful experience a lot has been written, but generally focusing on the education and methodology applied to the period called "comprehensive school", which covers ten years from the moment children turn six.

This article tackles a practical experience through participation in the «Savonia UAS 2018 International Week», an event that brings together important university professors and staff from different universities around the world, with many European countries as participants. In this event and in follow up activities, the aim is to get to know a model of university education from the perspective of the Faculty of Business, Tourism and Hospitality at the Savonia University of Applied Sciences (UAS) and the possibilities offered to identify innovative teaching strategies that could be applied to the major in Sustainable Tourism Management at Universidad Estatal a Distancia (UNED) and future possibilities for interuniversity cooperation.

Key words: Finland, education, tourism, innovation.

\section{RÉSUMÉ}

\section{Apprentisage pour le futur: expérience en Filande}

Dans les dernières decennies, la Finlande a misé sur un modèle éducatif de plus haut niveau. Il ya plusieurs publications de cette expérience réussie, notamment de la perspective éducative et de la méthodologie mise en place dans le periode nommé «comprensive school», qui s'étale sur dix années à partir de 6 ans.

Cet article porte sur l'expérience pratique à travers de la participation dans la «Semaine Internationale» organisée par la SAVONIA, Université de Sciences Appliquées située en Finlande. Cet événement réunit à des enseignants réputés et des autorités universitaires de diverses universités du monde, avec une grande participation des universités européennes.

Dans le cadre de cette rencontre et des activités réalisées ultérieurement, on vise à connaître le modèle d'enseignement universitaire de la conception de la Faculté des Affaires, Turisme et Hospitalité de l' Université de Sciences Appliquées de Savonia (UAS) et les possibilités d'indeitifier 
des stratégies d'enseignement novatrices que puissent être appliquées dans la filière de Gestion Touristique Durable de L’Université de l'État à Distance (UNED) ainsi que des futures opportunités de coopération interuniversitaire.

Mots-clés: Finlande, enseignement, tourisme, innovation.

\section{Bibliografía}

Finish National Board of Education. «La educación finlandesa en síntesis». Savonia University of Applied Sciences. Course Catalogue, 2013.

Sitio web de Savonia University of Applied Sciences (2017). Acceso el 18 de mayo de 2018, http://portal.savonia.fi/amk/en

Välijärvi, Jouni (2008). Evaluación y pedagogía en la Escuela inclusiva finlandesa. Foro Educativo Nacional, Bogotá, Colombia. 